Authorship note: MD and JK contributed equally to this work.

Conflict of interest: LSM is a full-time employee of Janssen Pharmaceuticals and may hold Johnson \& Johnson stock and stock options. LSM received prior grant support from AstraZeneca, Pfizer, Boehringer Ingelheim, Regeneron Pharmaceuticals, and Moderna Therapeutics; was a paid consultant for Armirall and Janssen Research and Development; was on the scientific advisory board of Integrated Biotherapeutics; and is a shareholder of Noveome Biotherapeutics, all of which are developing therapeutics against infections (including Staphylococcus aureus and other pathogens) and/or inflammatory conditions.

Copyright: (c) 2021, Leyva-Castillo et al. This is an open access article published under the terms of the Creative Commons Attribution 4.0 International License.

Submitted: March 25, 2021

Accepted: September 29, 2021

Published: November 8, 2021

Reference information: /CI Insight. 2021;6(21):e149953.

https://doi.org/10.1172/jici.

insight.149953.

\section{Basophil-derived IL-4 promotes cutaneous Staphylococcus aureus infection}

\author{
Juan-Manuel Leyva-Castillo, ${ }^{1,2}$ Mrinmoy Das, ${ }^{1,2}$ Jennifer Kane, ,,2 Maria Strakosha, ${ }^{1,2}$ Sonal Singh, ${ }^{1,2}$ \\ Daniel Sen Hoi Wong, ${ }^{1,2}$ Alexander R. Horswill, ${ }^{3}$ Hajime Karasuyama, ${ }^{4}$ Frank Brombacher, ${ }^{5}$ \\ Lloyd S. Miller, ${ }^{6}$ and Raif S. Geha ${ }^{1,2}$ \\ 'Division of Immunology, Boston Children's Hospital, Boston, Massachusetts, USA. ²Department of Pediatrics, Harvard \\ Medical School, Boston, Massachusetts, USA. ${ }^{3}$ Department of Immunology and Microbiology, University of Colorado \\ School of Medicine, Aurora, Colorado, USA. ${ }^{4}$ Inflammation, Infection and Immunity Laboratory, TMDU Advanced Research \\ Institute, Tokyo Medical and Dental University (TMDU), Tokyo, Japan. International Center for Cenetic Engineering and \\ Biotechnology Cape Town Component and Health Science Faculty, University of Cape Town, Cape Town, South Africa. \\ ${ }^{6}$ Department of Dermatology, Johns Hopkins University School of Medicine, Baltimore, Maryland, USA.
}

Superficial cutaneous Staphylococcus aureus (S. aureus) infection in humans can lead to soft tissue infection, an important cause of morbidity and mortality. IL-17A production by skin TCR $\gamma \delta^{+}$cells in response to IL-1 and IL- 23 produced by epithelial and immune cells is important for restraining $S$. aureus skin infection. How $S$. aureus evades this cutaneous innate immune response to establish infection is not clear. Here we show that mechanical injury of mouse skin by tape stripping predisposed mice to superficial skin infection with $S$. aureus. Topical application of $S$. aureus to tape-stripped skin caused cutaneous influx of basophils and increased /14 expression. This basophil-derived IL-4 inhibited cutaneous IL-17A production by TCR $\gamma \delta^{+}$cells and promoted S. aureus infection of tape-stripped skin. We demonstrate that IL-4 acted on multiple checkpoints that suppress the cutaneous IL-17A response. It reduced I/1 and II23 expression by keratinocytes, inhibited IL-1+IL-23-driven IL-17A production by TCR $\gamma \delta^{+}$cells, and impaired IL-17A-driven induction of neutrophil-attracting chemokines by keratinocytes. IL-4 receptor blockade is shown to promote I/17a expression and enhance bacterial clearance in tape-stripped mouse skin exposed to $S$. aureus, suggesting that it could serve as a therapeutic approach to prevent skin and soft tissue infection.

\section{Introduction}

Staphylococcus aureus ( $S$. aureus) is the most common causative agent of bacterial skin and soft tissue infections (SSTIs) (1-3). Impetigo, a superficial skin infection that commonly affects children, can lead to SSTIs. $S$. aureus commonly infects the skin of healthy individuals following trauma, burns, or surgical interventions, as well as the skin of patients with chronic diseases characterized by itching, such as atopic dermatitis (AD) and diabetes, suggesting that skin barrier disruption predisposes to cutaneous $S$. aureus infection (3-6).

IL-17A is a proinflammatory cytokine produced by CD $4^{+}$Th17 cells, TCR $\gamma \delta^{+}$cells, and type 3 innate lymphoid cells (ILC3s). IL-17A plays an important role in protection against mucosal infections, by recruiting neutrophils and promoting the production of antimicrobial peptides (AMPs) by epithelial cells $(7,8)$ Patients and mice with defects in IL-17A, IL-17A receptor chains, or IL-17A signaling, or defects in neutrophil number or function, are susceptible to mucosal and skin infections caused by $S$. aureus (9-11). In particular, IL-17A production by TCR $\gamma \delta^{+} \mathrm{T}$ cells and subsequent neutrophil recruitment to the skin play a critical role in controlling cutaneous $S$. aureus infection $(7,8,11-14)$. The cytokines IL-1 and IL-23 are produced by several cells in the skin, including keratinocytes, DCs, and macrophages, and play an important role in driving IL-17A production $(15,16)$. Patients and mice with defects in IL-1 and IL-23 production or signaling have defective IL-17A production and are susceptible to $S$. aureus infections (16-22).

Basophils account for less than $1 \%$ of peripheral blood leukocytes. Basophils produce IL- 4 and IL-13 and have been identified as important players in type 2 immune responses against allergens and parasitic infection (23). IL-4 has been shown to inhibit IL-17A production via STAT6 inhibition to the Il17a promoter (24). Basophils may promote Th17 responses by secreting IL-6 (25-28). Moreover, basophil-derived 
TNF- $\alpha$ enhances survival in sepsis induced by cecal ligation and puncture in mice (29). It is not known whether basophils and their products play a role in cutaneous $S$. aureus infection.

The mechanisms by which $S$. aureus circumvents the innate immune response to establish infection in injured skin are not well understood. We show that tape stripping, which only disrupts the upper layers of the epidermis, rendered mouse skin susceptible to $S$. aureus infection and that IL- 4 derived from basophils recruited into $S$. aureus-exposed, tape-stripped skin inhibited cutaneous IL-17A production and promoted $S$. aureus skin infection. We further demonstrate that IL-4 inhibited Illb and Il23 expression in keratinocytes, acted on TCR $\gamma \delta^{+}$cells to inhibit their IL-17A production in response to IL-1 $\beta$ and IL-23, and blocked IL-17A-driven induction of neutrophil-attracting chemokines in keratinocytes. Furthermore, we show that IL-4 signaling blockade reversed the susceptibility of tape-stripped skin to $S$. aureus infection.

\section{Results}

Mechanical skin injury promotes superficial cutaneous infection by $S$. aureus. We examined the clearance of topically applied $S$. aureus from shaved skin without or with tape stripping. Tape stripping resulted in a significant increase in transepidermal water loss (TEWL) that persisted for 72 hours (Figure 1A), demonstrating effective disruption of the skin barrier. A total of $1 \times 10^{8}$ colony-forming units (CFU) of community-acquired methicillin-resistant $S$. aureus (MRSA; parental strain USA300 SF8300) labeled with the fluorescent dye PSVue 794 were applied topically. The SF8300 MRSA strain used in this study was the most prevalent $S$. aureus strain causing infections in the United States in the early 2000s, is one of the better characterized MRSA strains, and is the one most commonly used in laboratory studies (30-32). Persistence of $S$. aureus was determined at $0,24,48$, and 72 hours by in vivo fluorescence imaging, as well as by measuring the numbers of CFU in skin homogenates. $S$. aureus was virtually completely cleared from shaved skin 24 hours after its application, as evidenced by the extinction of PSVue 794 fluorescence (Figure 1B) and by the recovery of negligible numbers of CFU at 24 hours and none at 48 and 72 hours (Figure 1C). In contrast, the decay in PSVue 794 fluorescence was significantly slower after application of $S$. aureus to tape-stripped skin, persisting throughout 48 hours (Figure 1B). Moreover, the numbers of CFU recovered at 24 hours were more than half those applied on day 0 , and viable $S$. aureus bacteria were consistently recovered at 48 and 72 hours (Figure 1C).

Histologic examination of skin sections taken on day 3 revealed that tape stripping caused epidermal hyperplasia that was further increased by application of $S$. aureus (Figure 1D). Importantly, Gram staining revealed the presence of Gram-positive bacteria in the epidermis of tape-stripped, but not shaved, skin exposed to $S$. aureus (Figure 1E). Immunofluorescence examination on day 3 following topical application of GFP-expressing, community-acquired MRSA strain USA300 LAC revealed the presence of $\mathrm{GFP}^{+}$bacteria in the epidermis of tape-stripped skin but not shaved skin (Figure 1F). Taken together, these results indicate that mechanical injury of skin promotes superficial cutaneous infection with $S$. aureus.

$I L-17 A$ from $T C R \gamma \delta^{+}$cells restrains $S$. aureus infection of mechanically injured skin. IL-17A is important for the clearance of intradermally injected $S$. aureus $(8,14)$. We examined the IL-17A response to superficial skin infection with $S$. aureus. Il17a expression was not detectable in shaved mouse skin but was minimally induced by tape stripping (Figure 2A). S. aureus application modestly upregulated Il17a expression in shaved skin (Figure 2A). In contrast, it caused a drastic increase in 1117 a expression in tape-stripped skin (Figure 2A). S. aureus application to shaved skin caused minimal or no increase in the expression of the neutrophil-attracting chemokines Cxcl1, Cxcl2, and Cxcl3; modest neutrophil infiltration; and no detectable increase in the expression of the AMP-encoding genes cathelicidin AMP (Camp) and defensin beta 14 (Defb14) (Figure 2, B-D; and Supplemental Figure 1, A and B; supplemental material available online with this article; https://doi.org/10.1172/jci. insight.149953DS1). Cxcl1, Cxcl2, and Cxcl3 expression; neutrophil infiltration; as well as Camp, Defb1, Defb3, Defb4, and Defb14 expression increased modestly after tape stripping but increased markedly after application of $S$. aureus to tape-stripped skin (Figure 2, B-D; and Supplemental Figure 1, A and B). These results indicate that $S$. aureus infection of mechanically injured skin causes a robust local IL-17A and AMP induction.

To investigate whether IL-17A restrains superficial skin infection by $S$. aureus, we examined $I l 17 a^{-1-}$ mice. Skin fluorescence 24 hours following application of PSVue 794-labeled S. aureus to tape-stripped skin was significantly higher in $I l 17 a^{-/}$mice compared with WT controls (Figure 2E). Moreover, the numbers of $S$. aureus CFU recovered from skin homogenates on day 3 were significantly higher in $I l 17 a^{-1-}$ mice compared with WT controls (Figure 2F). As expected, cutaneous expression of Cxcl1, Cxcl2, Cxcl3; neutrophil infiltration; and expression of Camp, Defb4, and Defb14 after S. aureus application to tape-stripped skin were all significantly reduced in $1117 a^{-/-}$mice compared with WT controls (Figure 2, G-I, and Supplemental Figure 1C). 

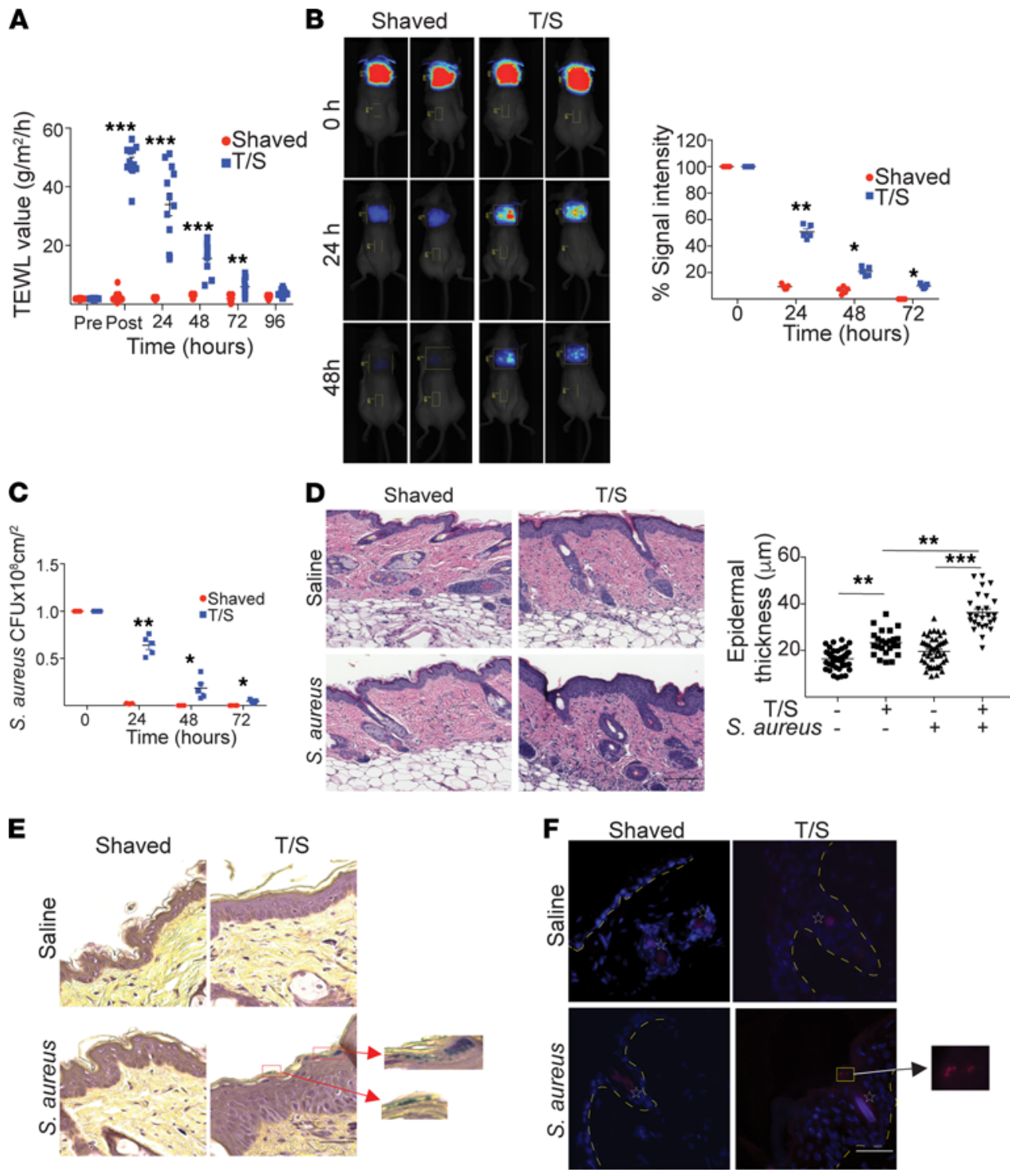

Figure 1. Mechanical skin injury promotes superficial cutaneous infection by S. aureus. (A) Effect of tape stripping on TEWL. (B) Representative in vivo fluorescence imaging (left) and quantitative analysis of the decay in PSVue 794 fluorescence (right) following topical application of $1 \times 10^{8}$ PSVue 794-labeled S. aureus strain USASF8300 to shaved and non-tape-stripped or shaved and tape-stripped (T/S) skin of BALB/c mice. (C) Numbers of colony forming units (CFU) in skin homogenates following application of $1 \times 10^{8}$ PSVue 794-labeled S. aureus strain USASF8300 to shaved or T/S skin of BALB/c mice. (D) Representative H\&E staining (left) and quantitation of epidermal thickness of shaved or T/S skin sections from BALB/c mice 72 hours after application of saline or PSVue 794-labeled S. aureus strain USASF8300. Scale bar: $100 \mu \mathrm{m}$. (E) Gram staining of skin sections from the same experiment as in $\mathbf{D}$. Red squares highlight Gram-positive bacteria. Scale bar: $50 \mu \mathrm{m}$. (F) Representative fluorescence images of sections of shaved or T/S skin of BALB/c mice 72 hours after application of saline or GFP-labeled S. aureus LAC strain. Yellow square highlights GFP+ bacteria; stars indicate autofluorescent hair follicles. Scale bar: $50 \mu \mathrm{m}$. Results in A-D are representative of 2 independent experiments with 4-5 mice/group. ${ }^{*} P<0.05,{ }^{*} P<0.005,{ }^{* *} P<0.001$ by 1 -tailed Student's $t$ test (A-C) or 1-way ANOVA (D).

TCR $\gamma \delta^{+}$cells, CD $4^{+}$TCR $\alpha \beta^{+}$Th17 cells, and ILC3s produce IL-17A (7). Tcrd ${ }^{-1}$ mice, which lack $\mathrm{TCR} \gamma \delta^{+}$cells, but have normal numbers of TCR $\alpha \beta^{+}$cells and ILC3s, failed to upregulate cutaneous $1117 a$ expression and had decreased neutrophil influx and higher loads of $S$. aureus following $S$. aureus application to tape-stripped skin (Figure 2, J-L). In addition, intracellular flow cytometry demonstrated that TCR $\gamma \delta^{+}$ cells but not $\mathrm{CD}^{+} \mathrm{T}$ cells or ILC3s isolated from $S$. aureus-infected skin were the main source of IL-17A produced after PMA and ionomycin stimulation (Supplemental Figure 2). These results indicate the importance of IL-17A produced by TCR $\gamma \delta^{+}$cells in clearing $S$. aureus infection from mechanically injured skin. 
A

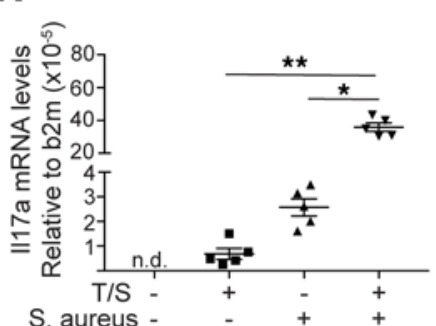

B

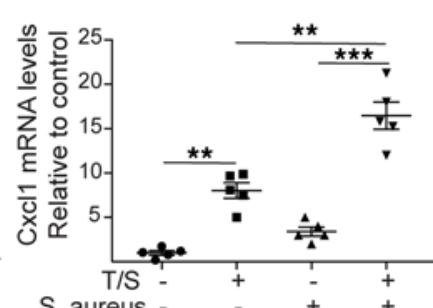

C

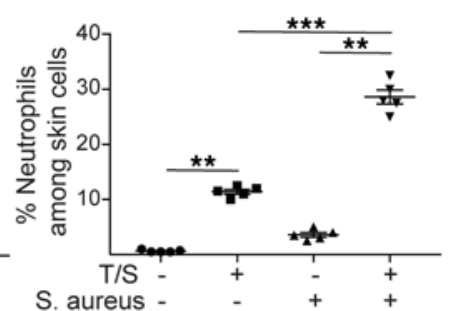

D

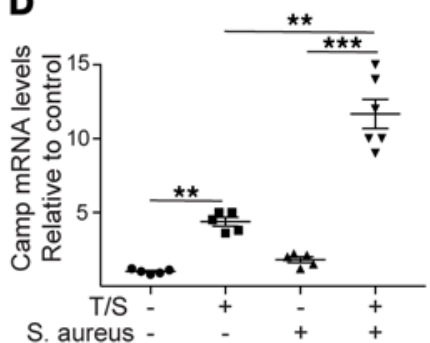

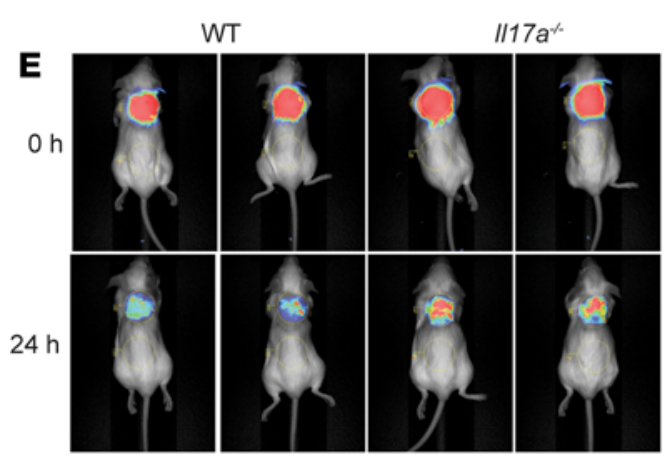

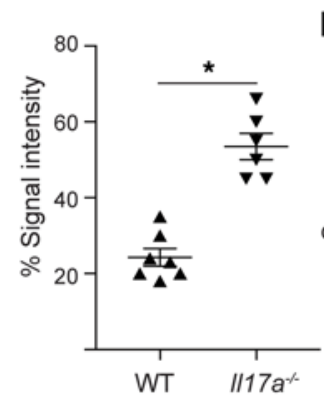

$\mathbf{F}$
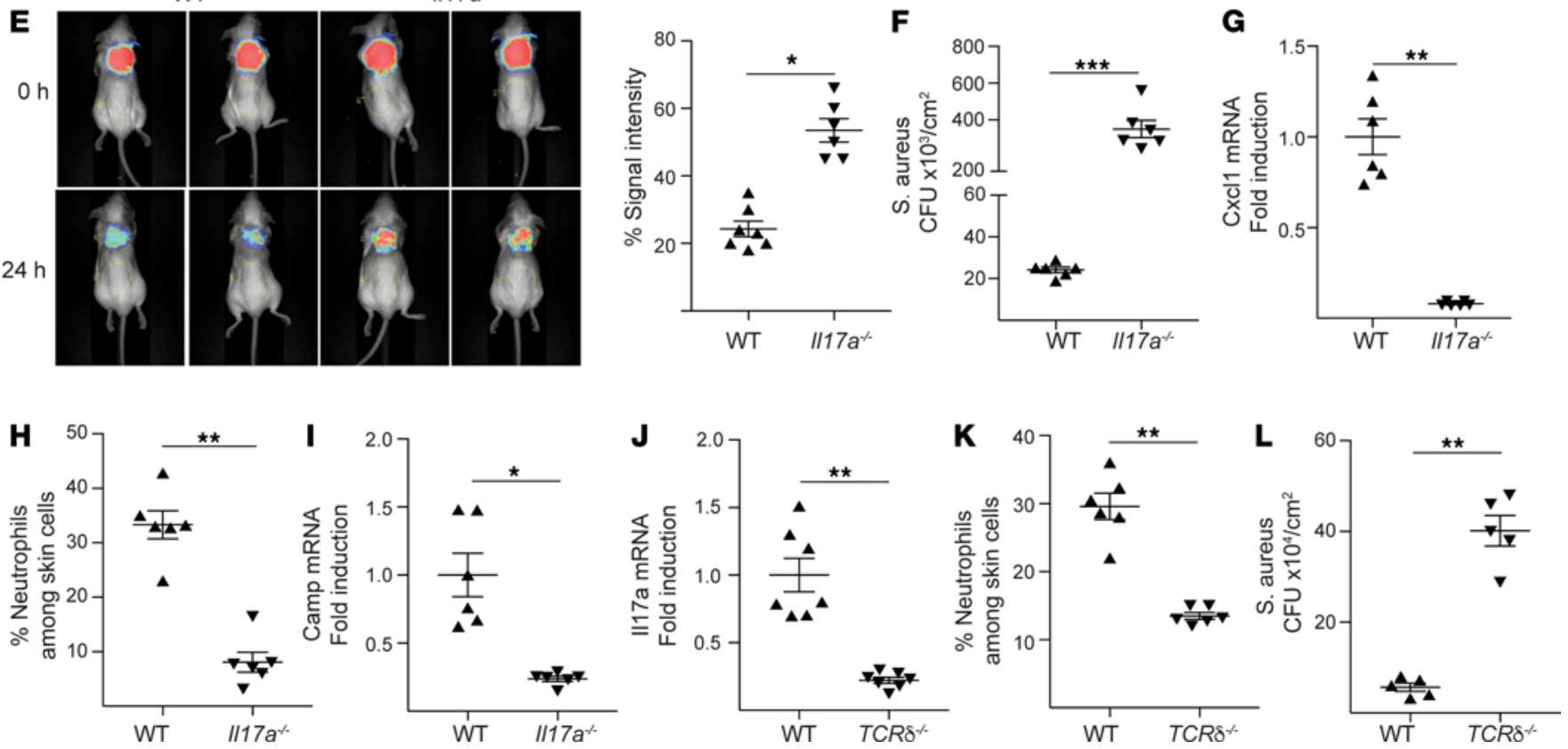

Figure 2. IL-17A from TCR $\gamma \boldsymbol{\delta}^{+}$cells protects mechanically injured skin from superficial infection by S. aureus. (A-D) II17a expression (A), Cxc/7 expression (B), percentage of neutrophils (C), and Camp expression (D) in non-tape-stripped or tape-stripped (T/S) skin of BALB/C mice 24 hours after application of S. aureus (+) or saline (-) control. (E) Representative in vivo fluorescence imaging (left) and quantitative analysis of the PSVue 794 fluorescence 0 and 24 hours (right) following topical application of $1 \times 10^{8}$ PSVue 794 labeled S. aureus strain USASF8300 to shaved and tape stripped (T/S) skin of $/ 17 a^{-1-}$ mice and WT controls. (F) Numbers of CFU in skin homogenates 72 hours following application of $1 \times 10^{8} \mathrm{~S}$. aureus strain USASF8300 to T/S skin of $/ 17 a^{-/-}$mice and WT controls. (G-I) Cxc/1 expression (G), percentage of neutrophils among skin cells $(\mathbf{H})$, and Camp expression (I) in T/S skin of $/ 117 a^{-1-}$ mice and WT controls 24 hours following application of $1 \times 10^{8}$ S. aureus to T/S skin of $/ 117 a^{-/-}$mice and WT controls. (J-L) II17a expression (J) and neutrophil infiltration (K) at 24 hours and numbers of CFU (L) at 72 hours in T/S skin from Tcrd ${ }^{-1-}$ mice and WT controls following application of $1 \times 10^{8} \mathrm{~S}$. aureus. n.d., not detected. Results in A-L are representative of 2 independent experiments with 4-5 mice/group. ${ }^{*} P<0.05,{ }^{*} P<0.005,{ }^{* * *} P<0.001$ by 1 -way ANOVA (A-D) or 1-tailed Student's $t$ test (E-L). b2m, beta-2-microglobulin.

IL-1 and IL-23 promote cutaneous Il17a expression in mechanically injured skin infected by S. aureus. The cytokines IL-1 and IL-23 drive IL-17A production by TCR $\gamma \delta^{+}$cells (16). Expression of Illa, Illb, and Il23p19 in tape-stripped skin markedly increased following application of $S$. aureus (Figure 3A). Keratinocytes express predominantly Illa while both keratinocytes and myeloid cells express Illb and Il23p19(33-37). Illa expression in tape-stripped skin exposed to $S$. aureus was confined predominantly to the epidermis. In contrast, $I l 1 b$ was expressed predominantly in the dermis. Il23p19 was expressed in both layers but significantly more in epidermis than dermis (Figure 3B). Upregulation of Il23p19 expression in tape-stripped skin exposed to $S$. aureus was virtually abolished in $I l 1 r 1^{-1-}$ mice (Figure 3C). Moreover, recombinant IL-1 $\alpha$ (rIL-1 $\alpha$ ) induced Il23p19 expression in epidermal sheets from WT mice (Figure 3D), suggesting an autocrine loop was involved in IL-23 production by keratinocytes.

The role of IL-1 and IL-23 in inducing a protective IL-17A response in S. aureus-infected, mechanically injured skin was examined in $I l 1 r 1^{-/-}$mice and following IL-23 blockade in WT mice, respectively. Cutaneous $I 117 a$ expression, neutrophil infiltration, and $S$. aureus clearance were significantly decreased in $I l 1 r 1^{1^{-1}}$ mice compared with WT controls as well as in mice treated with neutralizing anti-IL-23p19 antibody compared with mice treated with IgG isotype control (Figure 3, E and F). These results indicate that both IL-1 and IL-23 play an important role in driving IL-17A production by $\mathrm{TCR} \gamma \delta^{+}$cells during superficial skin infection. 
A
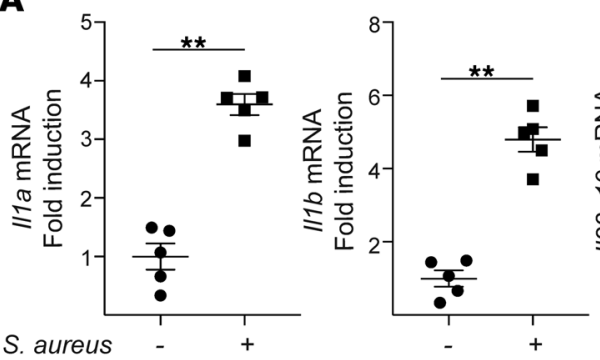

B

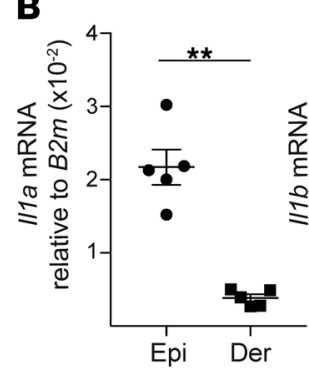

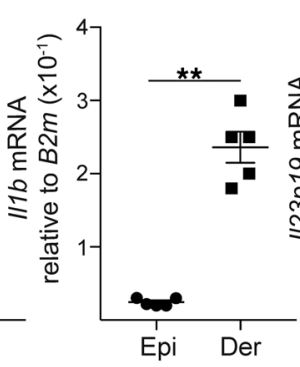

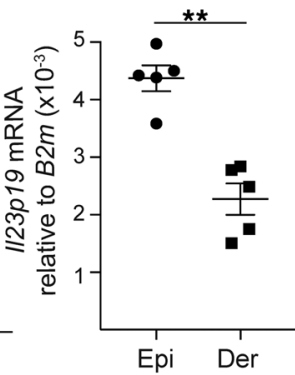

c

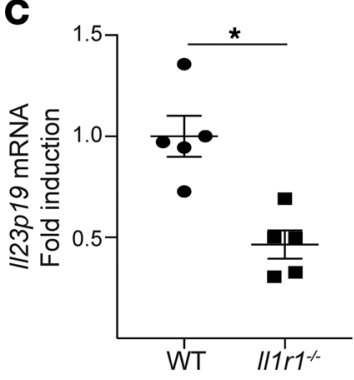

D

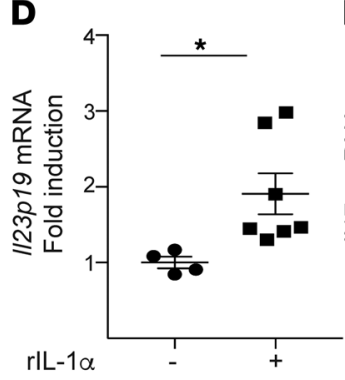

E
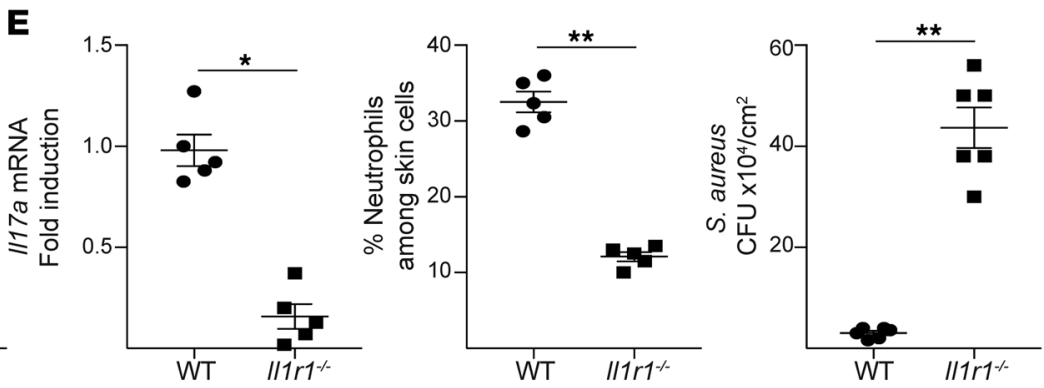

$\mathbf{F}$
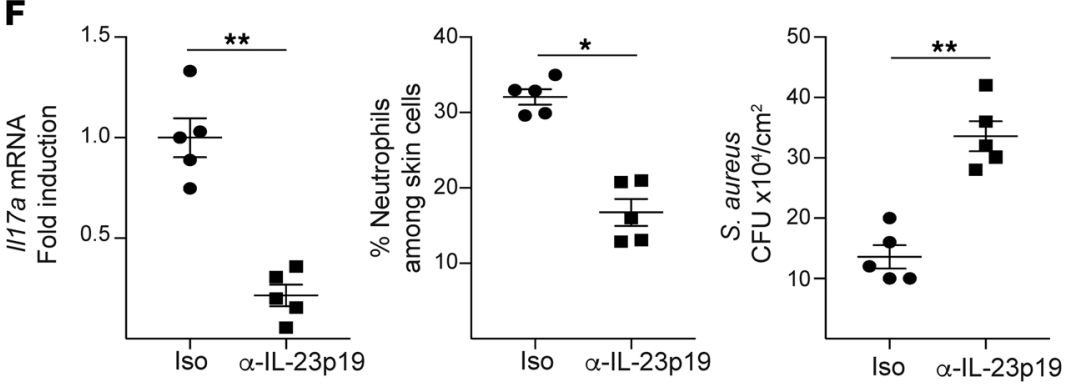

Figure 3. Epidermal cell-derived IL-1 and IL-23 promote cutaneous II17a expression in mechanically injured skin infected by S. aureus. (A and B) I/1a, I/1b, and II23p19 expression in full-thickness skin (A) and epidermal and dermal sheets (B) 24 hours following application of $1 \times 10^{8} \mathrm{~S}$. aureus to tape-stripped (T/S) skin of BALB/c mice. (C) I/23p19 expression in T/S skin 24 hours following topical application of $1 \times 10^{8} \mathrm{~S}$. aureus in I/1r1 ${ }^{-/-}$mice and WT controls.

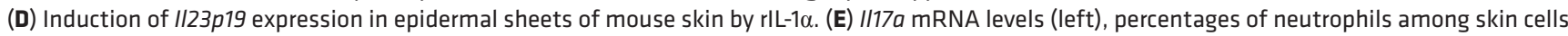
(middle), and numbers of CFU (right) 72 hours following application of $1 \times 10^{8} \mathrm{~S}$. aureus to T/S skin of $/ 11 \mathrm{r}^{-/-}$mice and WT controls. (F) I/17a mRNA levels (left), percentages of neutrophils among skin cells (middle), and numbers of CFU (right) 72 hours following application of $1 \times 10^{8} \mathrm{~S}$. aureus to T/S skin of WT BALB/c mice treated with anti-IL-23p19 antibody or isotype controls. Results in A-F are representative of 2 independent experiments with 4-5 mice/ group. ${ }^{*} P<0.05,{ }^{* *} P<0.005$ by 1 -tailed Student's $t$ test.

Basophil-derived IL-4 suppresses cutaneous Il17a expression and promotes $S$. aureus infection of mechanically injured skin. IL-4 impairs the induction and maintenance of adaptive Th17 immune responses (36). We investigated whether IL-4 suppresses the TCR $\gamma \delta^{+}$cell-dependent cutaneous IL-17A response that protects tape-stripped skin from superficial infection with $S$. aureus. Expression of Il4 was minimal in shaved skin and did not increase following application of $S$. aureus (Figure 4A). Tape stripping significantly increased Il4 expression in the skin; exposure to $S$. aureus increased it further (Figure 4A). Cutaneous expression of Il17a and of the chemokine Cxcl1 and of the AMP Camp following application of $S$. aureus to tape-stripped skin was significantly increased in $I 4^{-1-}$ mice compared with WT controls (Figure 4, B-D). Importantly, the numbers of $S$. aureus $\mathrm{CFU}$ recovered from skin homogenates after $S$. aureus application to tape-stripped skin were significantly decreased in $I l 4^{--}$mice compared with WT controls (Figure 4E).

Basophils are an important source of innate cell-derived IL-4 (38). Flow cytometry analysis of skin cell suspensions revealed that $\mathrm{CD}^{-} 5^{+} \mathrm{CD} 3^{-} \mathrm{IgE}^{+} \mathrm{CD} 117^{-}$basophils accounted for less than $1 \%$ of $\mathrm{CD}^{-} 5^{+} \mathrm{CD}^{-}$ cells in shaved mouse skin before and after application of $S$. aureus. This percentage significantly increased 24 hours after tape stripping and markedly increased following application of $S$. aureus to tape-stripped skin (Figure $4 \mathrm{~F}$ ). In contrast, tape stripping and $S$. aureus application caused minimal changes in the percentages of $\mathrm{IgE}^{+} \mathrm{CD} 117^{+}$mast cells in the skin (Figure $4 \mathrm{~F}$ ). To investigate whether basophils are a major source of 
A

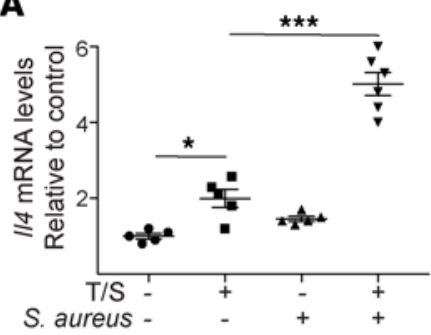

B

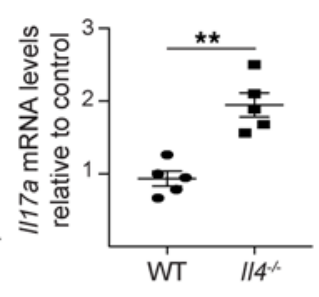

C

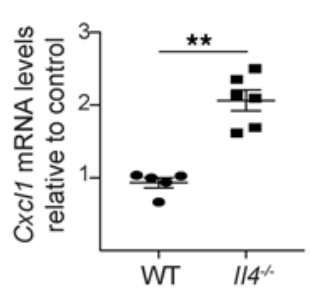

D

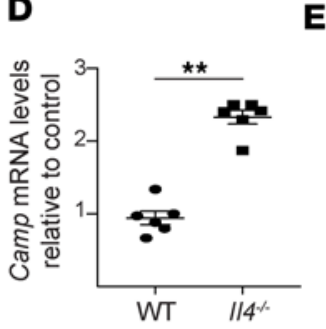

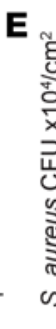

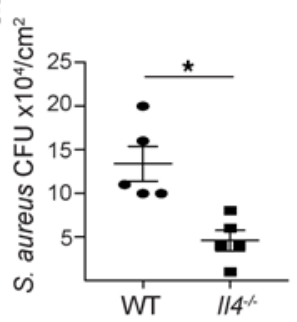

F Gated on $\mathrm{CD} 45^{+} \mathrm{CD} 3$-live cells

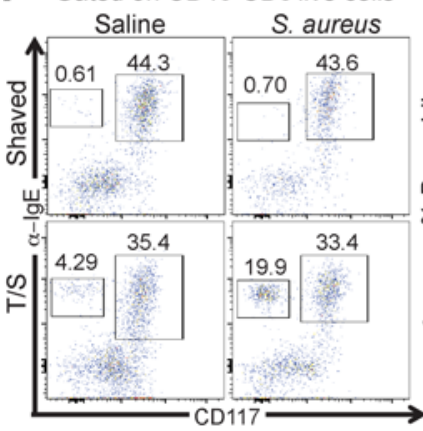

G

H

I

J

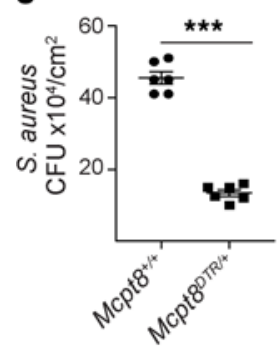

N

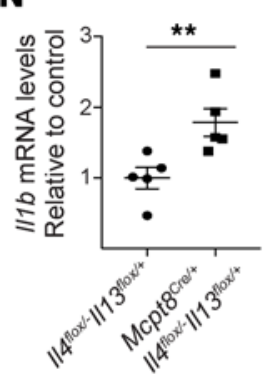

K

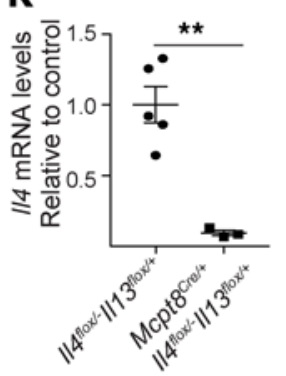

$\mathbf{L}$

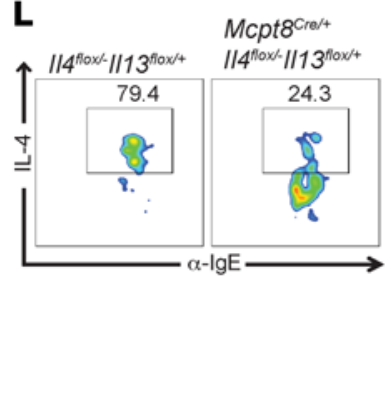

o

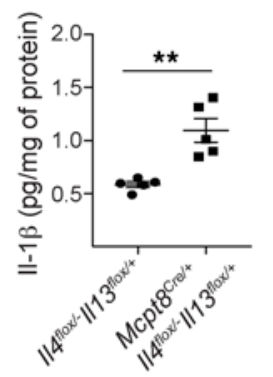

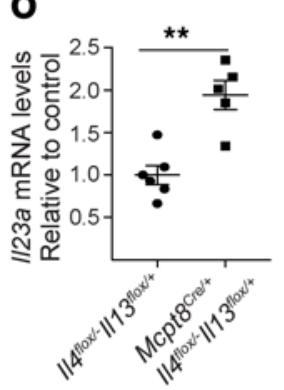
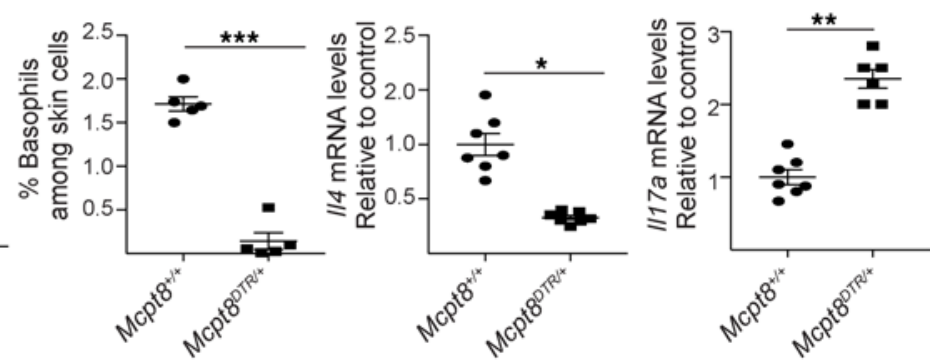

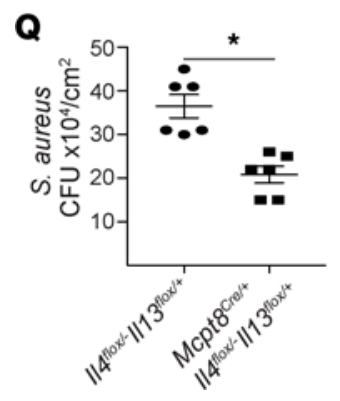

Figure 4. Basophil-derived IL-4 suppresses cutaneous II17a expression and promotes S. aureus superficial infection of mechanically injured skin. (A) Cutaneous $/ / 4$ expression 24 hours following topical application of $1 \times 10^{8} \mathrm{~S}$. aureus to shaved or shaved and tape-stripped (T/S) skin of BALB/C mice. (B-E) Cutaneous expression of I/17a (B), Cxc/1 (C), and Camp (D) at 24 hours, and numbers of CFU (E), 72 hours following topical application of S. aureus to T/S skin from $/ 14^{-/-}$ mice and WT controls. (F) Representative flow cytometry analysis of CD117 and Fc\&RI expression by CD45+CD3- cells (left) and quantitation of $C D 45^{+}{ }^{+} D 3^{-} F c \varepsilon R I^{+} C D 117^{-}$basophils (right) in shaved and shaved and T/S skin of BALB/c mice 24 hours after application of S. aureus. (C) Quantitation of CD45+CD3-FC\&RI+CD117- basophils in T/S skin of DT-treated Mcpt8 ${ }^{\text {DTR/+ }}$ and $\mathrm{Mcpt}^{+/+}$controls 24 hours after topical application of S. aureus. (H-J) //4 expression (H) and I/17a expression (I) at 24 hours and numbers of CFU (J) in T/S skin of DT-treated Mcpt8 $8^{\text {DTR/+ }}$ and $\mathrm{Mcpt}^{+/+}$controls 72 hours after topical application of S. aureus. (K-P) //4 expression (K), representative flow cytometry analysis of IL-4-producing basophils (left) and quantitation (right) (L), levels of I/1a mRNA (left) and IL-1 $\alpha$ protein (right) (M), levels of I/1b mRNA (left) and IL-1 $\alpha$ (right) (N), II23 mRNA (left) and IL-23 (right) levels (0), and levels of II17a mRNA (left) and IL-17A (right) (P) at 24 hours, and numbers

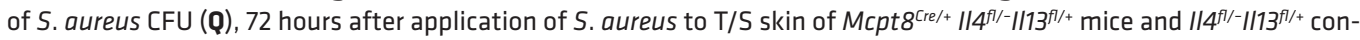
trols. Results in A-P are representative of 2 independent experiments with 4-5 mice/group. ${ }^{*} P<0.05,{ }^{* *} P<0.005$, ${ }^{* * *} P<0.001$ by 1-way ANOVA ( $\mathbf{A}$ and $\mathbf{F}$ ) or 1-tailed Student's $t$ test (B-E and $\mathbf{H}-\mathbf{Q}$ ). 
IL-4 in tape-stripped skin, $M c p t 8^{D T R /+}$ mice, which express diphtheria toxin receptor (DTR) selectively in basophils (39), and their $\mathrm{Mcpt}^{+/+}$littermates were treated with DT. The percentage of basophils in tapestripped skin exposed to $S$. aureus was drastically decreased in DT-injected Mcpt ${ }^{D T R /+}$ mice compared with DT-injected $\mathrm{Mcpt}^{+/+}$controls (Figure 4G). Importantly, Il4 expression in tape-stripped skin sites exposed to $S$. aureus was significantly diminished in DT-injected $M c p t 8^{D T R /+}$ mice compared with controls (Figure $4 \mathrm{H})$. In contrast, Il17a expression at these sites was significantly higher in DT-injected Mcpt8DTR/+ mice compared with controls (Figure 4I). Importantly, the numbers of CFU recovered from skin homogenates after $S$. aureus application to tape-stripped skin were significantly reduced in DT-injected $M c p t 8^{D T R /+}$ mice compared with controls (Figure 4J).

To investigate the role of basophil-derived IL- 4 in $S$. aureus colonization of mechanically injured skin, we generated Mcpt frel+ $^{\text {ch }} I 4^{f /-} 13^{f+}$ mice with selective ablation of IL-4 in basophils. To create this line, we crossed

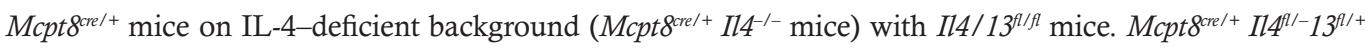
mice, but not $I l 4^{f /+} 13^{f /+}$ control littermates, failed to upregulate cutaneous $I l 4$ expression after application of $S$. aureus to tape-stripped skin (Figure $4 \mathrm{~K}$ ). In addition, basophils from Mcpt8cre/+ $I l 4^{f /-} 13^{f /+}$ mice were severely impaired in their ability to produce IL-4 compared with basophils from $I l 4^{f /+} 13^{f /+}$ control littermates (Figure 4L). In contrast, cutaneous IL-1 $\alpha$, IL-1 $\beta$, and IL-23 mRNA and protein levels at these sites were significantly increased in $M c p t 8^{c r e /+} I l 4^{f /-} 13^{f /+}$ mice compared with controls (Figure 4, M-O). In addition, Mcpt$8^{c r e /+} I l 4^{f /-} 13^{f /+}$ mice exhibited increased cutaneous IL-17A production, due to the increased percentage of IL-17A ${ }^{+} \mathrm{TCR} \gamma \delta^{+}$cells infiltrating their infected skin (Figure 4P). Importantly, the numbers of $S$. aureus CFU recovered from skin homogenates after $S$. aureus application to tape-stripped skin were significantly reduced in $M c p t 8^{\text {crel+ }} I l 4^{f /-} 13^{f /+}$ mice compared with controls (Figure 4Q). Together, these results indicate that IL-4 derived from basophils recruited to mechanically injured skin promotes cutaneous infection with $S$. aureus.

$I L-4$ acts at multiple checkpoints to inhibit the protective cutaneous $I L-17 A$ response against superficial infection by $S$. aureus. Mechanical injury induced by tape stripping promotes the production of damage-associated molecular patterns and cytokines. These include TNF- $\alpha$ and hyaluronic acid (HA), which drive the expression of IL-1 $\alpha$ and IL-23, respectively, in keratinocytes $(33,40)$. Keratinocytes express type II IL-4 receptor (IL-4R). The type II IL-4R binds both IL- 4 and IL-13 and shares the IL-4R $\alpha$ chain with the type I IL-4R, which binds IL-4 but not IL-13. Addition of rIL-4 inhibited TNF- $\alpha$-driven Il1a expression as well as HA-driven Il23p19 expression by epidermal sheets from WT mice but not $\mathrm{Ilr} 4 \mathrm{a}^{-1-}$ mice (Figure 5, A and B). These in vitro findings suggested that IL-4 could inhibit cutaneous Il17a expression in response to $S$. aureus exposure by down-

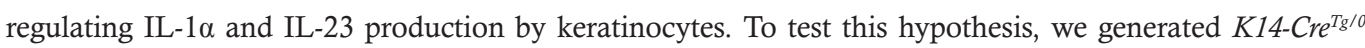
$I l 4 \mathrm{ra}^{f l /}$ - mice, which lack IL-4R $\alpha$ selectively in keratinocytes. Cutaneous expression of Illa, Il23p19, and Il17a following $S$. aureus application to tape-stripped skin was significantly increased in $\mathrm{K} 14-\mathrm{Cre}^{\mathrm{Tg} / 0} \mathrm{Il}_{4 \mathrm{ral}}{ }^{\mathrm{fl}-}$ mice compared with $\mathrm{K} 14-\mathrm{Il}_{4} \mathrm{r}^{\mathrm{fl}-}$ controls (Figure 5, C-E). Importantly, the numbers of CFU recovered from skin homogenates after $S$. aureus application to tape-stripped skin were significantly reduced in $\mathrm{K} 14-\mathrm{Cre}^{\mathrm{Tg} / 0} \mathrm{Il}_{\mathrm{rat}} \mathrm{gl}^{f /}$ mice compared with controls (Figure 5F).

$\mathrm{TCR} \gamma \delta^{+}$cells, the major producers of IL-17A in mechanically injured skin exposed to $S$. aureus, express type I IL-4R (41). We investigated whether IL-4 has a direct effect on Il17a expression by TCR $\gamma \delta^{+}$cells following stimulation with IL-1 and IL-23. rIL-4 markedly inhibited IL-1 $\beta+$ IL-23-driven Il17a expression by

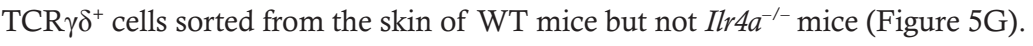

Keratinocytes are a major target for IL-17A-driven expression of neutrophil-attracting chemokines (42). We examined whether IL-4 has a direct effect on IL-17A-driven expression of the neutrophil chemoattractant $C x c 11$ by keratinocytes. rIL-4 inhibited IL-17A-driven expression of the neutrophil-attracting chemokine Cxcl1 by epidermal sheets from WT mice but not Ilr4a ${ }^{-1-}$ mice (Figure $5 \mathrm{H}$ ). The above results suggest that IL-4 may act at multiple checkpoints to inhibit the protective cutaneous IL-17A response of mechanically injured skin to $S$. aureus and consequently promote infection.

$I L-4 R \alpha$ blockade protects mechanically injured skin from superficial $S$. aureus infection. IL-4R $\alpha$ blockade by $\mathrm{mAb}$ is approved for human use. We investigated whether IL-4R $\alpha$ blockade could protect against $S$. aureus infection of mechanically injured skin. Mice were administered rat anti-mouse IL-4R $\alpha-$ blocking mAb or IgG isotype control 2 hours prior to application of $S$. aureus to tape-stripped skin. Cutaneous expression of Illa, Il23p19, and Il17a as well as neutrophil infiltration were significantly higher in $S$. aureus-exposed, tapestripped skin of recipients of the IL-4R $\alpha$-blocking antibody compared with recipients of IgG isotype control (Figure 6, A and B). However, there were no significant changes in Il4 mRNA levels or in the percentages of $\mathrm{TCR} \gamma \delta^{+}$cells and basophils following IL-4R $\alpha$ antibody blockade (Supplemental Figure 3). Importantly, 
A
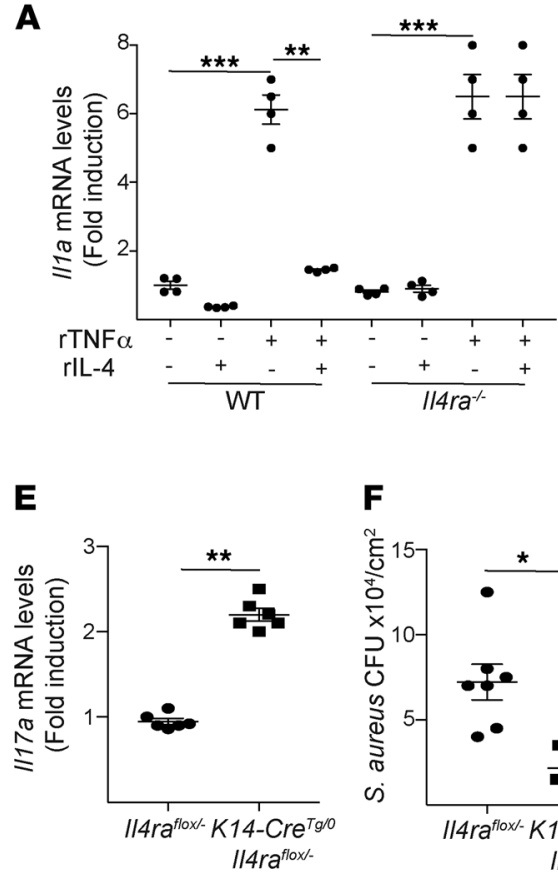
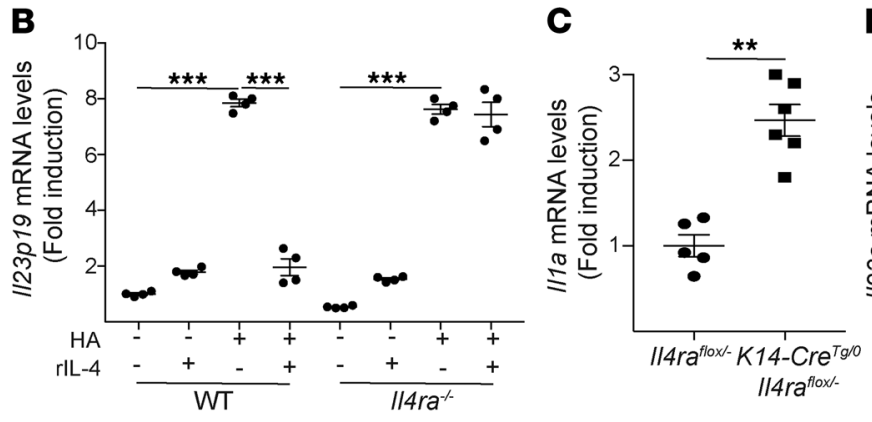

D

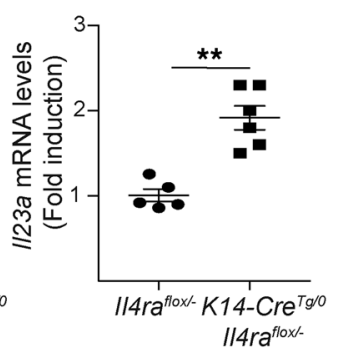

Figure 5. IL-4 acts at multiple checkpoints to inhibit the protective cutaneous IL-17A response against superficial infection by S. aureus. (A and B) Effect of rlL-4 on the induction of $/ 17 a$ by TNF- $\alpha$ (A) and $/ 123$ p19 by HA (B) in epidermal sheets from $/ 14 \mathrm{ra}^{-/-}$mice and WT controls. (C-F) Cutaneous expression of

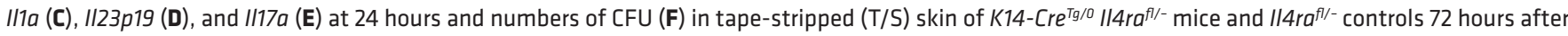
topical application of $1 \times 10^{8} \mathrm{~S}$. aureus. (C) Effect of IL-4 on IL-1 $\beta+\mathrm{IL}-23$-driven induction of $/ 117 a$ expression in TCR $\gamma \delta^{+}$cells from $/ 14 \mathrm{ra}^{-/-}$mice and WT controls. (H) Effect of IL-4 on IL-17A-driven induction of Cxc/7 expression in epidermal sheets from $/ 14 \mathrm{ra}^{-/-}$mice and WT controls. Results in $\mathbf{A}-\mathbf{H}$ are representative of 2 independent experiments with 4-5 mice/group. ${ }^{*} P<0.05,{ }^{* *} P<0.005,{ }^{* * *} P<0.001$ by 1-way ANOVA (A, B, G, and $\left.\mathbf{H}\right)$ or 1-tailed Student's $t$ test (C-F).

IL-4R $\alpha$ blockade significantly reduced the numbers of $S$. aureus CFU recovered from the skin (Figure 6C). These results indicate that IL-4 signaling blockade restrains $S$. aureus infection of mechanically injured skin.

\section{Discussion}

We have uncovered an important role for basophil-derived IL-4 in promoting superficial skin infection with $S$. aureus. Our results demonstrate that basophils are recruited following exposure of $S$. aureus on tapestripped skin and that basophil-derived IL-4 acts at multiple checkpoints to inhibit the IL-17A response of $\mathrm{TCR} \gamma \delta^{+} \mathrm{T}$ cells that protects against infection. Importantly, we show that IL-4R $\alpha$ blockade, currently in use for the treatment of patients with $\mathrm{AD}$, protects tape-stripped skin from superficial $S$. aureus infection.

Shaved but otherwise intact mouse skin was resistant to the establishment of superficial skin infection by $S$. aureus. In contrast, mouse skin mechanically injured by tape stripping was permissive for $S$. aureus superficial skin infection. Like intradermal and subcutaneous infection with $S$. aureus, superficial $S$. aureus skin infection elicited a TCR $\gamma \delta^{+}$cell-dependent protective local IL-17A response that promoted neutrophil recruitment and AMP production, both of which are important for the clearance of $S$. aureus $(7,8,14,43)$. IL-1 and IL-23, which may be derived in part from keratinocytes, were required for the induction of IL-17A following $S$. aureus infection of mechanically injured skin, consistent with the important role of these 2 cytokines in the induction of IL-17A in inflammation, infections, and wound healing $(16,18,19,34)$. IL-1 signaling was required for the optimal induction of cutaneous $I l 23 p 19$ expression in skin superficially infected with $S$. aureus. This is in line with a recent report indicating that recombinant IL-1R antagonist decreases Il23p19 expression and neutrophil recruitment into the skin induced by ablation of corneodesmosin (44).

We have shown that basophils are recruited to mechanically injured skin exposed to $S$. aureus and partially block the IL-17A/neutrophil recruitment axis that protects against superficial skin infection. In agreement with this result, acute depletion of basophils exacerbates mouse models of experimental autoimmune encephalomyelitis (EAE) and colitis mediated by IL-17A and neutrophils $(26,28,45)$. Other studies have shown that basophils promote type 17 immune responses by producing IL- 6 and that chronic depletion of basophils exacerbates the lupus phenotype in MRL-lpr/lpr mice and the severity of EAE in WT mice (25, 
A

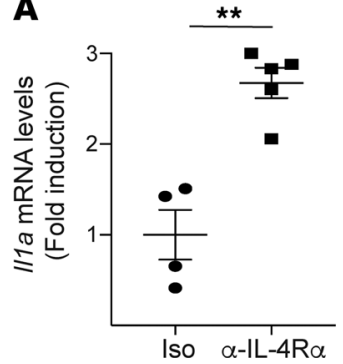

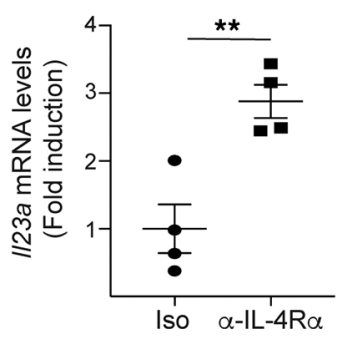

B

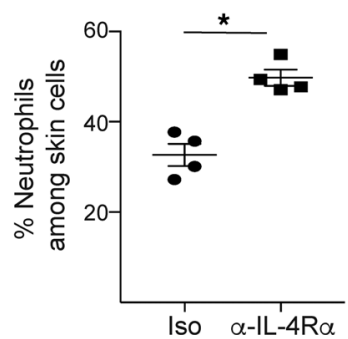

C

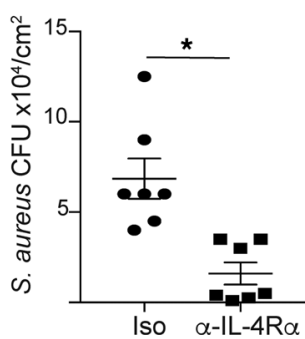

Figure 6. IL-4R blockade protects mechanically injured skin from superficial S. aureus infection. (A-C) Cutaneous expression of I/1a, II23p19, and I/17a (A); neutrophil infiltration in the skin (B) at 24 hours; and numbers of CFU (C) 72 hours after topical application of $1 \times 10^{8}$ S. aureus strain to tape-stripped (T/S) skin of WT recipients of anti-IL-4R $\alpha$ antibody or IgG isotype control. Results in A-C are representative of 2 independent experiments with 4-5 mice/ group. ${ }^{*} P<0.05,{ }^{* *} P<0.005$ by 1 -tailed Student's $t$ test.

27). Thus, basophils may play a context-dependent dual role in the in vivo IL-17 response, initially suppressing its induction, then once it is established, supporting its maintenance.

Basophils are a key source of IL-4 under steady-state as well as pathological conditions (38). Our results demonstrate that basophils are the main source of IL-4 in skin superficially infected with $S$. aureus and that basophil-derived IL-4 dampens the protective IL-17A response against infection. This inhibition is in line with the known role of IL-4 in suppressing in type 17 responses. IL-4 suppresses IL-17A expression by $\mathrm{CD} 4^{+} \mathrm{T}$ cells as well as the in vitro production of the IL-17-inducing cytokines IL-23p19, IL-1 $\beta$, and IL-6 by DCs and keratinocytes $(24,36)$. In vivo, treatment with rIL-4 improves disease severity in mouse models of EAE and psoriasis, 2 disease models associated with a type 17 immune response $(36,46,47)$.

Keratinocytes have emerged as important players in cutaneous immune responses, as they respond to autocrine and paracrine cytokines by producing cytokines, chemokines, and AMP. We show that IL-4 suppresses Il1a, Il23p19, and Cxcl1 expression by epidermal sheets in response to in vitro stimulation with TNF- $\alpha$, HA, and IL-17A, respectively. Importantly, mice lacking IL-4R $\alpha$ selectively in keratinocytes demonstrated increased cutaneous expression of the cytokines Illa, Il23p19, and Ill7a in mechanically injured skin exposed to $S$. aureus. In line with these results, IL-4 inhibits TNF- $\alpha-$ as well as IFN- $\gamma-$ driven expression of AMP-encoding genes by keratinocytes (48). It also suppresses the production of IL-1 $\beta$ and the AMP hBD2 in epidermal cells from patients with psoriasis (49), who exhibit increased IL-4Ra expression by epidermal cells (50). Together, these results suggest that IL-4 signaling in keratinocytes inhibits cutaneous IL-17A induction and plays a counterpoint to IL-17 signaling in these cells, possibly in an attempt to restore homeostasis in inflamed skin.

The diversity of the skin microbiome and its interactions with pathogenic microorganisms shape the immune response and limit the growth of pathogens (51-53). We cannot exclude that perturbation of skin microbiome induced by tape stripping or in mice with deficiencies in cells or cytokines could affect the establishment of cutaneous $S$. aureus infection. Furthermore, different $S$. aureus strains produce different virulence factors and toxins that could alter the protective immune response differently than the $S$. aureus $\mathrm{SF} 8300$ strain we used.

Administration of dupilumab, a human $\mathrm{mAb}$ against IL-4R $\alpha$, to patients with $\mathrm{AD}$ ameliorates the disease and results in improved local and systemic disease markers, as well as a decrease in $S$. aureus colonization of the skin (54-57). It is difficult to dissect the exact mechanisms by which IL-4R $\alpha$ blockade reduces the $S$. aureus cutaneous burden in patients with $\mathrm{AD}$. We demonstrate that IL-4R $\alpha$ blockade increases Il17a expression and promotes bacterial clearance from the skin in our mouse model of acute superficial infection with $S$. aureus. This is of clinical relevance because it suggests that IL-4R blockade might represent an effective therapeutic approach to inhibit $S$. aureus skin colonization or prevent $S$. aureus SSTIs.

\section{Methods}

Mice. BALB/c and C57BL/6J WT mice were purchased from Charles River Laboratories. Rag2 $2^{-/}$mice were

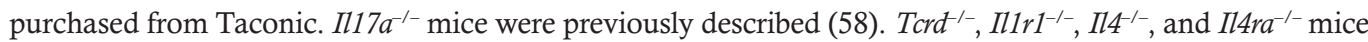
were purchased from The Jackson Laboratory. $I l 4 / 13^{f / l}$ mice were a gift of Andrew McKenzie (Medical Research Council, Laboratory of Molecular Biology, Cambridge, United Kingdom). Mcpt $8^{\text {DTR }}$ mice were a gift of TMDU and TMDU Advanced Research Institute, Tokyo, Japan. Il4ra ${ }^{\nexists / f l}$ mice was a gift from International Center for Genetic Engineering and Biotechnology, University of Cape Town, and South Africa 
Medical Research Council, Cape Town, South Africa. Mcpt8cre/+ mice on C57BL/6J background were purchased from The Jackson Laboratory and crossed with $\mathrm{Il}^{-/-}$mice on BALB/c background for 8 generations, then crossed with $I l 4 / 13^{f / f l}$ mice in BALB/c background. K14-Cre ${ }^{T g / 0}$ mice on C57BL/6J background were purchased from The Jackson Laboratory and crossed with $I l 4 \mathrm{ra}^{-/-}$in BALB/c background for 9 generations, then crossed with $I l 4 \mathrm{ra}^{f / / f}$ mice in BALB/c background. All mice were kept in a pathogen-free environment.

Skin cell preparation and flow cytometry. Skin pieces $\left(1 \mathrm{~cm}^{2}\right)$ from unmanipulated, tape-stripped, or $S$. aureusinfected mice were obtained. Skin pieces were finely chopped using scissors after fat removal and digested for 90 minutes in media containing liberase (Roche) and DNAse II (MilliporeSigma), with continuous shaking at $37^{\circ} \mathrm{C}$. Digested skin homogenates were filtered, washed, and resuspended in PBS and used for flow cytometry. Cells were preincubated with Fcy receptor-specific blocking mAb (clone 2.4G2, BioLegend) and washed before staining with the following mAbs: Alexa Fluor 700-anti-CD45 (clone 30-F11, BioLegend), BV605-anti-CD11b (clone M1/70, BioLegend), FITC-anti-Gr1 (clone RB6-8C5, BioLegend), CD3-efluor 450 (clone 17A2, eBioscience), IgE-PE (RME-1, BioLegend), and CD117-APC (clone ACK2, eBioscience). Cells were analyzed on LSR Fortessa (BD Biosciences), and the data were analyzed with FlowJo software.

Quantitative reverse transcription PCR. At indicated times after tape stripping or $S$. aureus infection, total skin and epidermal and dermal sheet RNA was extracted with Total RNA Isolation Kit (Ambion). cDNA was prepared with iScript cDNA Synthesis Kit (Bio-Rad). PCR reactions were run on an ABI Prism 7300 (Applied Biosystems) sequence detection system platform. TaqMan primers and probes were obtained from Life Technologies. The housekeeping gene $\beta_{2}$-microglobulin was used as an internal control.

S. aureus and quantification of skin infection. The community-acquired MRSA USA300 SF8300 strain, a gift of Binh Diep (UCSF, San Francisco, California, USA) and the GFP-producing USA300 LAC strain (59) were cultured in tryptic soy broth as previously described. Briefly, a $S$. aureus inoculum was streaked onto a tryptic soy agar plate and grown overnight at $37^{\circ} \mathrm{C}$. Single colonies were picked and inoculated into a $5 \mathrm{~mL}$ tube containing tryptic soy broth and cultured overnight in a shaking incubator. The following morning, 1:50 dilution of bacterial suspension was inoculated in $5 \mathrm{~mL}$ of tryptic soy broth and cultured for another 2 hours. Bacterial concentrations were estimated by measuring absorbance at $600 \mathrm{~nm}$. The bacteria were concentrated to $10^{8} \mathrm{CFU} / 50 \mu \mathrm{L}$ of PBS and used for cutaneous infection. CFUs were verified by overnight culturing of inoculum on CHROMagar plates. To enumerate the bacterial load in vivo, $S$. aureus was labeled with the PSVue794 reagent kit (LI-COR), following manufacturer's instructions. PSVue794 fluorescence was quantified at different time points using Pearl Trilogy Small Animal Imaging System (LI-COR). To enumerate the bacterial load from the skin, two $8 \mathrm{~mm}^{2}$ skin biopsies were obtained. After mechanical homogenization, serial dilutions of skin homogenates were cultured overnight on CHROMagar plates. The growth of USA300 strain was quantified by counting only pink colonies.

DTR-mediated cell depletion. Mcpt8 ${ }^{\text {DTR }}$ mice received an i.p. injection of DT (750 ng per $20 \mathrm{~g}$ body weight) 2 days before $S$. aureus application to tape-stripped skin.

$I L-23$ and IL-4R $\alpha$ blockade. Mice were injected i.v. with $100 \mu \mathrm{g}$ of rat monoclonal anti-mouse IL-23p19 antibody (Bio X Cell) or with $100 \mu \mathrm{g}$ of rat monoclonal anti-mouse IL-4R $\alpha$ antibody (BD Biosciences) 2 hours before $S$. aureus application to tape-stripped skin.

Preparation of epidermal and dermal sheets and culture. Ears from mice were split into dorsal and ventral halves, then floated for 30 minutes at $37^{\circ} \mathrm{C}$ on PBS containing $4 \mathrm{mg} / \mathrm{mL}$ dispase (MilliporeSigma). Dermal and epidermal sheets were split and stored at $-80^{\circ} \mathrm{C}$ for RNA analysis. Epidermal sheets were cultured with overnight HA $(10 \mu \mathrm{g} / \mathrm{mL}), \mathrm{rIL}-1$ (10 ng/mL), rTNF- $\alpha(20 \mathrm{ng} / \mathrm{mL})$, rIL-17A (50 ng/mL), and/or rIL-4 (50 ng/mL).

Histology. For H\&E and Gram staining, skin specimens were fixed in 4\% PFA and embedded in paraffin. Sections $(5 \mu \mathrm{m})$ of skin were stained with $\mathrm{H} \& \mathrm{E}$ or Gram stained by the Rodent Histopathology Core at Dana-Farber/Harvard Cancer Center. For fluorescence microscopy, $10 \mu \mathrm{m}$ cryosections were fixed in 4\% PFA and permeabilized using BD Biosciences Cytoperm Permeabilization buffer, and then slides were mounted using mounting medium containing DAPI (ProLong Gold) from Thermo Fisher Scientific. Pictures were captured using EVOS cell imaging systems.

Statistics. Statistical significance was determined by 1-tailed Student's $t$ test or 1-way ANOVA on GraphPad Prism. All the figures display mean \pm SEM. A $P$ value less than 0.05 was considered statistically significant.

Study approval. All procedures performed on the mice were in accordance with and approved by the Animal Care and Use Committee of Boston Children's Hospital. 


\section{Author contributions}

JMLC and RSG conceived the project and supervised the experiments. JMLC, MD, JK, MS, SS, and DSHW performed, analyzed, and interpreted results of the experiments. ARH, HK, FB, and LSM contributed critical reagents or mice. JMLC and RSG wrote the manuscript.

\section{Acknowledgments}

This work was funded by the NIH/National Institute of Allergy and Infectious Diseases (NIAID) Atopic Dermatitis Research Network grant U19AI117673 and NIH grant AI113294-01A1. JMLC was supported by a NIAID T32 training grant (5T32AI007512-32), a postdoctoral fellowship from CONACYT (Mexico), and a Boston Children's Hospital OFD/BTREC/CTREC Faculty Career Development Fellowship. We thank Dana-Farber/Harvard Cancer Center in Boston, Massachusetts, USA, for the service provided by the Rodent Histopathology Core. Dana-Farber/Harvard Cancer Center is supported in part by an NIH/National Cancer Institute Cancer Center Support Grant (NIH 5 P30 CA06516).

Address correspondence to: Juan-Manuel Leyva-Castillo or Raif S. Geha, Boston Children's Hospital, Division of Immunology, One Blackfan Circle, Boston, Massachusetts 02115, USA. Phone: 617.919.2482; Email: Manuel.LeyvaCastillo@childrens.harvard.edu (JMLC). Email: Raif.Geha@childrens.harvard.edu (RSG). JK's present address is: Department of Dermatology and Warren Alpert Medical School at Brown University, Rhode Island Hospital, Providence, Rhode Island, USA. SS's present address is: Department of Neurology, Johns Hopkins University, Baltimore, Maryland, USA. DSHW's present address is: Department of Biochemistry, The University of British Columbia, Vancouver, British Columbia, Canada. LSM's present address is: Immunology, Janssen Research and Development, Spring House, Pennsylvania, USA.

1. Moran GJ, et al. Methicillin-resistant $S$. aureus infections among patients in the emergency department. N Engl J Med. 2006;355(7):666-674.

2. Hiramatsu K, et al. Multi-drug-resistant Staphylococcus aureus and future chemotherapy. J Infect Chemother. 2014;20(10):593-601.

3. Tong SY, et al. Staphylococcus aureus infections: epidemiology, pathophysiology, clinical manifestations, and management. Clin Microbiol Rev. 2015;28(3):603-661.

4. Geoghegan JA, et al. Staphylococcus aureus and atopic dermatitis: a complex and evolving relationship. Trends Microbiol. 2018;26(6):484-497

5. Breen JD, Karchmer AW. Staphylococcus aureus infections in diabetic patients. Infect Dis Clin North Am. 1995;9(1):11-24

6. Crum-Cianflone NF, et al. Increasing rates of community-acquired methicillin-resistant Staphylococcus aureus infections among HIV-infected persons. Int J STD AIDS. 2007;18(8):521-526.

7. Jin W, Dong C. IL-17 cytokines in immunity and inflammation. Emerg Microbes Infect. 2013;2(9):e60.

8. Cho JS, et al. IL-17 is essential for host defense against cutaneous Staphylococcus aureus infection in mice. J Clin Invest. 2010;120(5):1762-1773.

9. McDonald DR. TH17 deficiency in human disease. J Allergy Clin Immunol. 2012;129(6):1429-1435.

10. Rigby KM, DeLeo FR. Neutrophils in innate host defense against Staphylococcus aureus infections. Semin Immunopathol. 2012;34(2):237-259.

11. Molne L, et al. Role of neutrophil leukocytes in cutaneous infection caused by Staphylococcus aureus. Infect Immun. 2000;68(11):6162-6167.

12. Malhotra N, et al. IL-22 derived from $\gamma \delta$ T cells restricts Staphylococcus aureus infection of mechanically injured skin. $J$ Allergy Clin Immunol. 2016;138(4):1098-1107.

13. Verdrengh M, Tarkowski A. Role of neutrophils in experimental septicemia and septic arthritis induced by Staphylococcus aureus. Infect Immun. 1997;65(7):2517-2521.

14. Marchitto MC, et al. Clonal $\mathrm{V} \gamma 6^{+} \mathrm{V} \delta 4^{+} \mathrm{T}$ cells promote IL-17-mediated immunity against Staphylococcus aureus skin infection. Proc Natl Acad Sci U S A. 2019;116(22):10917-10926.

15. Martin B, et al. Interleukin-17-producing $\gamma \delta \mathrm{T}$ cells selectively expand in response to pathogen products and environmental signals. Immunity. 2009;31(2):321-330.

16. Sutton CE, et al. Interleukin-1 and IL-23 induce innate IL-17 production from $\gamma \delta \mathrm{T}$ cells, amplifying Th17 responses and autoimmunity. Immunity. 2009;31(2):331-341.

17. Sutton C, et al. A crucial role for interleukin (IL)-1 in the induction of IL-17-producing T cells that mediate autoimmune encephalomyelitis. J Exp Med. 2006;203(7):1685-1691.

18. Lubberts E. The IL-23-IL-17 axis in inflammatory arthritis. Nat Rev Rheumatol. 2015;11(7):415-429.

19. Gaffen SL, et al. The IL-23-IL-17 immune axis: from mechanisms to therapeutic testing. Nat Rev Immunol. 2014;14(9):585-600.

20. Fragoulis GE, et al. Therapeutic targeting of IL-17 and IL-23 cytokines in immune-mediated diseases. Annu Rev Med. 2016;67:337-353.

21. Ali A, et al. IL-1 Receptor antagonist treatment aggravates Staphylococcal septic arthritis and sepsis in mice. PLoS One. 2015;10(7):e0131645.

22. Mayer-Barber KD, Yan B. Clash of the cytokine titans: counter-regulation of interleukin-1 and type I interferon-mediated inflammatory responses. Cell Mol Immunol. 2017;14(1):22-35. 
23. Karasuyama H, et al. Multifaceted roles of basophils in health and disease. J Allergy Clin Immunol. 2018;142(2):370-380.

24. Cooney LA, et al. Sensitivity and resistance to regulation by IL-4 during Th17 maturation. J Immunol. 2011;187(9):4440-4450.

25. Hermann FJ, et al. Basophils inhibit proliferation of CD4+ T cells in autologous and allogeneic mixed lymphocyte reactions and limit disease activity in a murine model of graft versus host disease. Immunology. 2015;145(2):202-212.

26. Pan Q, et al. Basophil activation-dependent autoantibody and interleukin-17 production exacerbate systemic lupus erythematosus. Front Immunol. 2017;8:348.

27. Yuk CM, et al. Basophil-derived IL-6 regulates $\mathrm{T}_{\mathrm{H}} 17$ cell differentiation and CD4 T cell immunity. Sci Rep. 2017;7:41744.

28. Musio S, et al. Treatment with anti-FceRI $\alpha$ antibody exacerbates EAE and T-cell immunity against myelin. Neurol Neuroimmunol Neuroinflamm. 2017;4(3):e342.

29. Piliponsky AM, et al. Basophil-derived tumor necrosis factor can enhance survival in a sepsis model in mice. Nat Immunol. 2019;20(2):129-140.

30. Diep BA, et al. Complete genome sequence of USA300, an epidemic clone of community-acquired meticillin-resistant Staphylococcus aureus. Lancet. 2006;367(9512):731-739.

31. O'Hara FP, et al. Molecular characterization of Staphylococcus aureus in the United States 2004-2008 reveals the rapid expansion of USA300 among inpatients and outpatients. Microb Drug Resist. 2012;18(6):555-561.

32. Vitko NP, Richardson AR. Laboratory maintenance of methicillin-resistant Staphylococcus aureus (MRSA). Curr Protoc Microbiol. 2013; Chapter 9:Unit 9C.2

33. Yoon J, et al. IL-23 induced in keratinocytes by endogenous TLR4 ligands polarizes dendritic cells to drive IL-22 responses to skin immunization. J Exp Med. 2016;213(10):2147-2166.

34. Miller LS, et al. Inflammasome-mediated production of IL-1beta is required for neutrophil recruitment against Staphylococcus aureus in vivo. J Immunol. 2007;179(10):6933-6942.

35. Archer NK, et al. Injury, dysbiosis, and filaggrin deficiency drive skin inflammation through keratinocyte IL-1 $\alpha$ release. $J$ Allergy Clin Immunol. 2019;143(4):1426-1443.

36. Guenova E, et al. IL-4 abrogates T(H)17 cell-mediated inflammation by selective silencing of IL-23 in antigen-presenting cells Proc Natl Acad Sci U S A. 2015;112(7):2163-2168.

37. Cho JS, et al. Neutrophil-derived IL-1 $\beta$ is sufficient for abscess formation in immunity against Staphylococcus aureus in mice. PLoS Pathog. 2012;8(11):e1003047.

38. Yamanishi Y, Karasuyama H. Basophil-derived IL-4 plays versatile roles in immunity. Semin Immunopathol. 2016;38(5):615-622.

39. Wada T, et al. Selective ablation of basophils in mice reveals their nonredundant role in acquired immunity against ticks. $J C l i n$ Invest. 2010;120(8):2867-2875.

40. Kutsch CL, et al. Tumor necrosis factor-alpha induces interleukin-1 alpha and interleukin-1 receptor antagonist production by cultured human keratinocytes. J Invest Dermatol. 1993;101(1):79-85.

41. Bloodworth $\mathrm{MH}$, et al. STAT6 signaling attenuates interleukin-17-producing $\gamma \delta \mathrm{T}$ cells during acute Klebsiella pneumoniae infection. Infect Immun. 2016;84(5):1548-1555

42. Nograles KE, et al. Th17 cytokines interleukin (IL)-17 and IL-22 modulate distinct inflammatory and keratinocyte-response pathways. Br J Dermatol. 2008;159(5):1092-1102.

43. Das S, Khader S. Yin and yang of interleukin-17 in host immunity to infection. F1000Res. 2017;6:741.

44. Li J, et al. Counterregulation between thymic stromal lymphopoietin- and IL-23-driven immune axes shapes skin inflammation in mice with epidermal barrier defects. J Allergy Clin Immunol. 2016;138(1):150-161.

45. Wakahara K, et al. Human basophils interact with memory T cells to augment Th17 responses. Blood. 2012;120(24):4761-4771

46. Falcone $\mathrm{M}$, et al. A critical role for IL-4 in regulating disease severity in experimental allergic encephalomyelitis as demonstrated in IL-4-deficient C57BL/6 mice and BALB/c mice. J Immunol. 1998;160(10):4822-4830.

47. Hahn M, Ghoreschi K. The role of IL-4 in psoriasis. Expert Rev Clin Immunol. 2017;13(3):171-173.

48. Albanesi C, et al. IL-4 and IL-13 negatively regulate TNF-alpha- and IFN-gamma-induced beta-defensin expression through STAT-6, suppressor of cytokine signaling (SOCS)-1, and SOCS-3. J Immunol. 2007;179(2):984-992.

49. Onderdijk AJ, et al. IL-4 Downregulates IL-1 $\beta$ and IL-6 and induces GATA3 in psoriatic epidermal cells: route of action of a Th2 cytokine. J Immunol. 2015;195(4):1744-1752.

50. Prens E, et al. Increased expression of interleukin-4 receptors on psoriatic epidermal cells. Am J Pathol. 1996;148(5):1493-1502.

51. Scharschmidt TC. Establishing tolerance to commensal skin bacteria: timing is everything. Dermatol Clin. 2017;35(1):1-9.

52. Paller AS, et al. The microbiome in patients with atopic dermatitis. J Allergy Clin Immunol. 2019;143(1):26-35.

53. Byrd AL, et al. The human skin microbiome. Nat Rev Microbiol. 2018;16(3):143-155.

54. Hamilton JD, et al. Dupilumab improves the molecular signature in skin of patients with moderate-to-severe atopic dermatitis. $J$ Allergy Clin Immunol. 2014;134(6):1293-1300.

55. Guttman-Yassky E, et al. Dupilumab progressively improves systemic and cutaneous abnormalities in patients with atopic dermatitis. J Allergy Clin Immunol. 2019;143(1):155-172.

56. Beck LA, et al. Dupilumab treatment in adults with moderate-to-severe atopic dermatitis. N Engl J Med. 2014;371(2):130-139.

57. Callewaert C, et al. IL-4R $\alpha$ blockade by dupilumab decreases Staphylococcus aureus colonization and increases microbial diversity in atopic dermatitis. J Invest Dermatol. 2020;140(1):191-202.E7.

58. Nakae S, et al. Antigen-specific T cell sensitization is impaired in IL-17-deficient mice, causing suppression of allergic cellular and humoral responses. Immunity. 2002;17(3):375-387.

59. Pang YY, et al. agr-Dependent interactions of Staphylococcus aureus USA300 with human polymorphonuclear neutrophils. J Innate Immun. 2010;2(6):546-559. 